\title{
Aplicação da Lei de Benford nos números de casos confirmados de COVID-19 em diferentes países
}

\section{Benford's Law applied to COVID-19 confirmed case numbers in different countries}

\author{
Rhômulo Oliveira Menezes \\ Universidade Federal do Pará (UFPA), Instituto de Educação Matemática e Científica (IEMCI), \\ Programa de Pós-Graduação em Educação em Ciências e Matemáticas (PPGECM), Belém, PA, \\ Brasil \\ https://orcid.org/0000-0001-9042-8323, rhomulo.menezes4542@escola.seduc.pa.gov.br
}

\begin{abstract}
Informações do Artigo
Como citar este artigo

MENEZES, Rhômulo Oliveira. Aplicação da

Lei de Benford nos números de casos

confirmados de COVID-19 em diferentes

países. REMAT: Revista Eletrônica da

Matemática, Bento Gonçalves, RS, v. 7, n. 1,

p. e3005, 5 fev. 2021. DOl:

https://doi.org/10.35819/remat2021v7i1id4586
\end{abstract}

Histórico do Artigo

Submissão: 17 de setembro de 2020.

Aceite: 6 de dezembro de 2020.

\section{Palavras-chave}

COVID-19

Números de Casos Confirmados

Lei de Benford

Teste de Comparação de Frequências

\section{Resumo}

A Lei de Benford afirma que, em conjuntos de números aleatórios, a probabilidade de o primeiro dígito desses números ser 1 é maior do que a dos dígitos seguintes. Dessa forma, a distribuição proposta por essa lei mostra que o 1 tem aproximadamente $30,1 \%$ de chances de ser o primeiro; em seguida, o dígito 2, com 17,6\%; o 3, com $12,5 \%$ e assim por diante, até chegar ao dígito 9 , com $4,6 \%$ de chance. Nesse contexto, assume-se, como objetivo, verificar se a Lei de Benford se aplica aos números de casos confirmados da doença COVID-19 em diferentes países. A pesquisa, do tipo quantitativa, tratou e analisou dados coletados no site da Organização Mundial de Saúde (OMS) dos seguintes países, escolhidos aleatoriamente, conforme notoriedade nas mídias nacionais e internacionais: China, Itália, Nova Zelândia, Brasil e Estados Unidos da América (EUA). Para avaliar a discrepância entre as frequências relativas observadas e esperadas, utilizou-se o teste de comparação de frequências. Os resultados encontrados mostraram que os números da China e da Nova Zelândia tiveram $X^{2}$ calculado menor que o $X^{2}$ crítico, enquanto a Itália, o Brasil e os EUA tiveram $X^{2}$ calculado maior que o $X^{2}$ crítico, todos em um nível de significância de $5 \%$. Assim, concluiu-se que a Lei de Benford aplicou-se aos números de casos confirmados da doença COVID-19 na China e na Nova Zelândia, sendo rejeitada pelos números de casos confirmados da doença COVID-19 na Itália, no Brasil e nos EUA.

\section{Abstract}

Benford's Law states that in sets of random numbers the prospect of the first digit of these numbers being 1 is greater than that of the following digits. Thus, the distribution proposed by Benford's law shows that number 1 has approximately a $30.1 \%$ chance of being the first digit then the digit 2, with $17.6 \%$, digit 3 , with $12.5 \%$, and so on, until number 9 is reached, having a $4.6 \%$ chance. In this context, the objective of the study is to verify whether this law applies to the numbers of confirmed cases of COVID-19 in different countries. The research, employing a quantitative method, treated and analyzed data collected on the World Health Organization (WHO) website, selecting the following countries, chosen randomly, according to the notoriety received in national and international media: China, Italy, New Zealand, Brazil and the United States of America (USA). To assess the discrepancy between the observed and expected relative frequencies, the frequency comparison test was used. The results found demonstrated that the numbers for China and New Zealand had calculated $X^{2}$ smaller than the critical $X^{2}$, while Italy, Brazil and the USA had calculated $X^{2}$ greater than the critical $X^{2}$, all at a $5 \%$ significance level. Consequently, it was concluded that Benford's Law applied to the numbers of confirmed cases of COVID-19 

USA.

\section{Introdução}

A Lei de Benford - também conhecida como Lei do Primeiro Dígito, Lei de NewcombBenford ou Lei dos Números Anômalos - foi observada por Newcomb (1881), astrônomo e matemático do século XIX, que, ao manusear livros de tabelas logarítmicas, percebeu que as primeiras páginas apresentavam maior desgaste em relação ao uso do que as seguintes, fazendoo inferir que mais números usados começavam pelo dígito 1, ou seja, mantissas de menor valor ocorriam com maior frequência que mantissas de maior valor. No entanto, a observação de Newcomb (1881) foi demonstrada e difundida por Benford (1938), que a analisou em conjuntos de números oriundos de diferentes contextos não relacionados, indicando-a como uma lei de probabilidade geral de ampla aplicação.

Muitos foram os estudos realizados com o propósito de identificar dados fabricados a partir da distribuição da Lei de Benford. Cunha (2013), em seu estudo da arte, destacou vários trabalhos que evidenciam essas aplicações. Dentre eles, os estudos de Nigrini (1992, 2000, 2012) e suas descobertas a partir da hipótese de que dados contábeis seguiam a distribuição da Lei de Benford detectaram dados fraudulentos aleatórios em sete companhias de New York, nos quais poucos valores começavam com o número 1 e muitos começavam com o 6. Para Cunha (2013, p. 52), este autor "mostrou a aplicabilidade generalizada da lei e seus usos práticos para detectar fraudes, erros e outras anomalias".

Outra aplicação da Lei de Benford foi em dados macroeconômicos, como demonstrado no artigo de Rauch et al. (2011). Ao utilizar a Lei de Benford nos dados macroeconômicos dos países membros da União Europeia, os autores construíram um ranking dos 27 países membros, conforme a extensão do desvio encontrado, indicando a Grécia como o país que teve maior desvio. Essa suspeita de manipulação dos dados foi posteriormente confirmada pela própria Comissão Europeia. Assim, esse estudo "evidenciou a efetividade da Lei de Benford na detecção de irregularidades e manipulações de dados macroeconômicos" (CUNHA, 2013, p. 40).

Além de dados contábeis e macroeconômicos, outra aplicação realizada foi em dados de eleições. Mebane $(2006,2009)$ analisou dados das eleições iranianas de 2009. Diferentemente das aplicações supracitadas, o estatístico descobriu que a contagem de votos tendia a seguir a Lei de Benford no segundo dígito, e não no primeiro, como comumente usada, fazendo-o constatar anomalias que indicavam fortemente a ocorrência de fraude na vitória de Ahmadinejad no Iran.

Assim, neste trabalho, considerando a singularidade da Lei de Benford de mostrar probabilidades distintas para a ocorrência do primeiro dígito em um universo aleatório de números, e considerando o conjunto de números derivados da pandemia do novo coronavírus 
(SARS-COV-2), causador da doença COVID-19, assumimos como objetivo verificar se a Lei de Benford se aplica aos números de casos confirmados da doença COVID-19 em diferentes países.

O artigo foi organizado em quatro seções, a contar da introdução. Na segunda seção, descreveremos características da pesquisa realizada, evidenciando o tipo de pesquisa, a fonte de dados, bem como instrumentos de coleta e de análise dos dados. $\mathrm{Na}$ terceira seção, apresentaremos os resultados encontrados. Encerraremos o artigo na quarta seção, na qual apresentaremos conclusões.

\section{Metodologia}

Nesta seção, apresentamos aspectos metodológicos da pesquisa relatada neste artigo, destacando o tipo de pesquisa, a fonte de coleta dos dados analisados, bem como características da Lei de Benford e do método estatístico utilizado na validação dos dados analisados.

\subsection{Tipo de pesquisa e fonte de coleta dos dados analisados}

Pesquisas de cunho quantitativo "consistem essencialmente em encontrar relações entre variáveis, fazer descrições recorrendo ao tratamento estatístico de dados recolhidos, testar teorias" (CARMO; FERREIRA, 2008, p. 196). Dessa forma, entendemos a pesquisa relatada neste artigo como sendo do tipo quantitativo, na medida em que buscamos verificar a Lei de Benford nos números confirmados de COVID-19 em diferentes países.

Os países foram selecionados de forma arbitrária, de acordo com a notoriedade que ganhavam com a proliferação da doença COVID-19 em seus territórios, como especificado a seguir: China, primeiros casos confirmados no mundo; Itália, um dos países da Europa mais afetados; Nova Zelândia, país referência no enfretamento e no controle de casos; Brasil, país mais afetado na América do Sul; e EUA, país que lidera os rankings como um dos mais afetados no mundo.

A coleta aconteceu entre os dias 12 e 16 de setembro de 2020, considerando o intervalo de meses janeiro-agosto, no site da Organização Mundial de Saúde (OMS ${ }^{1}$ ), no qual acessamos os números diários de casos confirmados de COVID-19 na China, na Itália, na Nova Zelândia, no Brasil e nos EUA.

\subsection{A Lei de Benford}

A Lei de Benford é dada por

$$
P(n)=\log _{10}\left(1+\frac{1}{n}\right)
$$

\footnotetext{
${ }^{1}$ Disponível em: https://covid19.who.int/. Acesso em: 12 e 16 de set. de 2020.
} 
em que $n \in[1,9]$ e $\mathrm{P}(\mathrm{n})$ referem-se à probabilidade de ocorrência do primeiro dígito $n$ em um universo de números aleatórios, como assinalado na Tabela 1.

Tabela 1 - Lei de Benford para o primeiro dígito.

\begin{tabular}{ccc}
\hline Dígito $(\boldsymbol{n})$ & $\begin{array}{c}\text { Probabilidade } \\
\text { do Primeiro } \\
\text { Dígito } \boldsymbol{P}(\boldsymbol{n})\end{array}$ & Percentual \\
\hline 1 & 0,301 & $30,1 \%$ \\
2 & 0,176 & $17,6 \%$ \\
3 & 0,125 & $12,5 \%$ \\
4 & 0,097 & $9,7 \%$ \\
5 & 0,079 & $7,9 \%$ \\
6 & 0,067 & $6,7 \%$ \\
7 & 0,058 & $5,8 \%$ \\
8 & 0,051 & $5,1 \%$ \\
9 & 0,046 & $4,6 \%$ \\
\hline
\end{tabular}

Fonte: Adaptado de Benford (1938, p. 556).

Na Figura 1 é possível observar o comportamento dos dados gerados com o modelo matemático da Lei de Benford (Tabela 1) no gráfico:

Figura 1 - Gráfico do percentual da frequência do primeiro dígito.

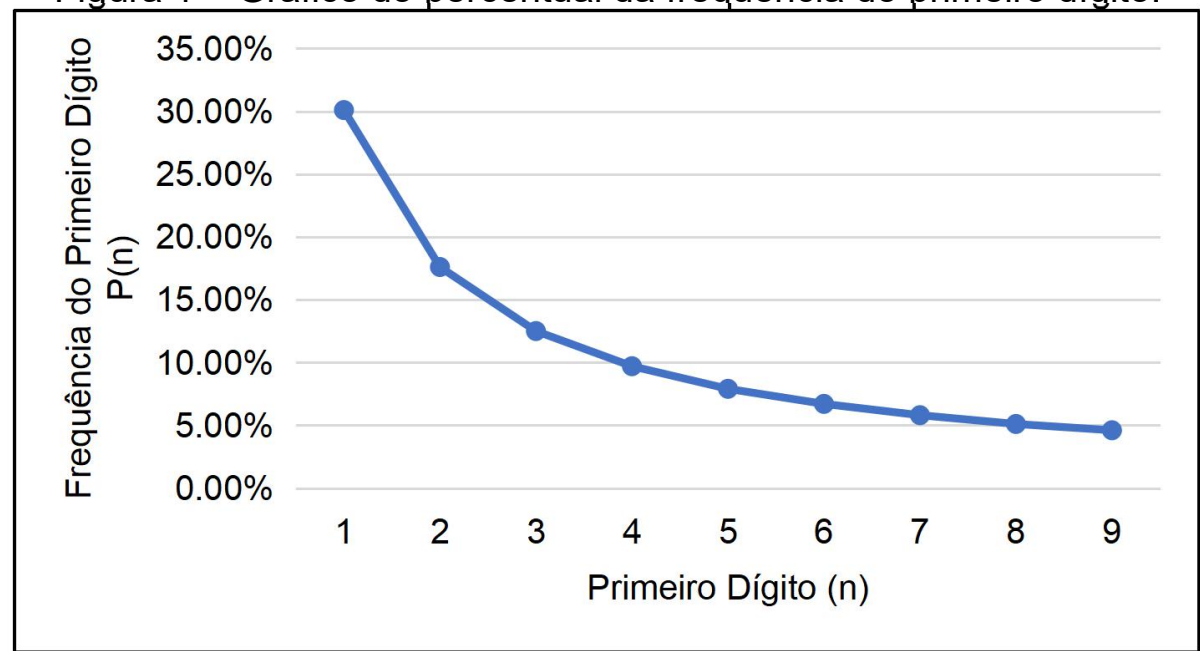

Fonte: Elaboração do autor (2020).

\subsection{Teste de comparação de frequências $\left(X^{2}\right)$}

Para o estudo deste artigo, considerando que foram investigadas amostras de números derivadas dos casos confirmados da doença COVID-19 em alguns países (Frequências observadas), segundo a Lei de Benford, com os percentuais para a frequência do primeiro dígito desses números (Frequências esperadas), utilizamos o teste de comparação de frequências. De acordo com Campos, Wodewotzki e Jacobini (2013), os resultados obtidos por meio de amostras nem sempre coincidem com os resultados teóricos esperados. Por exemplo, ao lançar uma moeda honesta 100 vezes, teoricamente estima-se que se pode esperar 50 vezes resultando cara e 50 vezes resultando coroa; no entanto, muitas vezes esse resultado não é obtido. 
Com o teste de comparação de frequências seria possível comparar, por exemplo, se o total de números de casos de COVID-19 de um determinado país (Frequências observadas) que começam com o dígito 1 difere de modo significativo do percentual ajustado pela Lei de Benford (Frequências esperadas), que no caso de o primeiro dígito ser 1, é de 30,1\% de chance. Essa medida de discrepância existente entre as frequências observadas e as frequências esperadas é dada pela estatística $X^{2}$, que é expressa por:

$$
X^{2}=\frac{\left(o_{1}-e_{1}\right)^{2}}{e_{1}}+\frac{\left(o_{2}-e_{2}\right)^{2}}{e_{2}}+\ldots+\frac{\left(o_{k}-e_{k}\right)^{2}}{e_{k}}=\sum \frac{\left(o_{j}-e_{j}\right)^{2}}{e_{j}} .
$$

De acordo com Campos, Wodewotzki e Jacobini (2013, p. 102), o processo do teste de comparação de frequências acontece da seguinte forma:

As frequências esperadas são calculadas com base em uma hipótese $H_{0}$. Se, para essa hipótese, o valor de $X^{2}$ calculado for maior que o valor crítico (dado em tabela), concluímos que as frequências observadas diferem de modo significativo das esperadas e rejeitamos $H_{0}$ ao nível de significância correspondente. No caso contrário, devemos aceitá-la ou, pelo menos, não rejeitá-la.

Para este estudo, consideramos, para o $X^{2}$ calculado, o somatório das nove possibilidades da Lei de Benford. Dessa forma, para o primeiro dígito temos $j \in\{1,2,3,4,5,6,7,8,9\}$, com grau de liberdade igual a $8(g l=9-1=8)$, expresso por

$$
X^{2}=\sum_{j=1}^{9} \frac{\left(o_{j}-e_{j}\right)^{2}}{e_{j}} .
$$

E, para o $X^{2}$ crítico, fixamos o nível de significância em $5 \%$, delimitando para este estudo os valores utilizados para o teste de comparação de frequências:

Tabela 2 - Valores considerados para o teste de comparação de frequências.

\begin{tabular}{ccc}
\hline $\mathbf{X}^{2}$ calculado & $\begin{array}{c}\text { Grau de } \\
\text { liberdade (gl) }\end{array}$ & $\mathbf{X}^{\mathbf{2}}$ crítico $^{2}$ \\
\hline$X^{2}=\sum_{j=1}^{9} \frac{\left(o_{j}-e_{j}\right)^{2}}{e_{j}}$ & 8 & 15,507 \\
\hline
\end{tabular}

Fonte: Elaboração do autor (2020).

Assim, para avaliar se a hipótese $H_{0}$ deste estudo (Lei de Benford aplicando-se aos números de casos confirmados de COVID-19 em diferentes países) foi rejeitada ou não, comparamos os valores de $X^{2}$ calculado e $X^{2}$ crítico, permitindo inferir duas opções:

$$
\begin{gathered}
\mathrm{X}^{2} \text { calculado } \geq \mathrm{X}^{2} \text { crítico } \rightarrow \text { rejeitar } \mathrm{H}_{0}, \\
\mathrm{X}^{2} \text { calculado }<\mathrm{X}^{2} \text { crítico } \rightarrow \text { não rejeitar } \mathrm{H}_{0} .
\end{gathered}
$$

\footnotetext{
2 Disponível em: https://www.ime.unicamp.br/ cnaber/Tabela\%20da\%20Qui-quadrado.pdf. Acesso em: 16 de set. de 2020.
} 


\section{Resultados}

Nesta seção, apresentamos os resultados obtidos, ao analisar as frequências relativas observadas no primeiro dígito dos números de casos confirmados de COVID-19 na China, na Itália, na Nova Zelândia, no Brasil e nos EUA. Os dados investigados referem-se aos números de casos confirmados de janeiro até agosto, de acordo com o surgimento dos primeiros casos em cada país.

\subsection{China}

Segundo o site de monitoramento da OMS, na China, de 4 de janeiro a 31 de agosto de 2020, houve 90.383 casos confirmados de COVID-19, conforme organizado na Tabela 3.

Tabela 3 - Números de casos diários confirmados de COVID-19 na China.

\begin{tabular}{|c|c|c|c|c|c|c|c|c|c|c|c|c|c|c|c|}
\hline \multicolumn{2}{|c|}{ Janeiro } & \multicolumn{2}{|c|}{ Fevereiro } & \multicolumn{2}{|c|}{ Março } & \multicolumn{2}{|c|}{ Abril } & \multicolumn{2}{|c|}{ Maio } & \multicolumn{2}{|c|}{ Junho } & \multicolumn{2}{|c|}{ Julho } & \multicolumn{2}{|c|}{ Agosto } \\
\hline Dia & $n .^{\circ}$ & Dia & $n .^{\circ}$ & Dia & $n .^{\circ}$ & Dia & $n .^{\circ}$ & Dia & $n .^{\circ}$ & Dia & $n .^{\circ}$ & Dia & $n^{\circ}$ & Dia & $n^{\circ}$ \\
\hline 1 & - & 1 & 2101 & 1 & 579 & 1 & 93 & 1 & 12 & 1 & 18 & 1 & 5 & 1 & 166 \\
\hline 2 & - & 2 & 2590 & 2 & 206 & 2 & 86 & 2 & 6 & 2 & 9 & 2 & 31 & 2 & 180 \\
\hline 3 & - & 3 & 2827 & 3 & 128 & 3 & 78 & 3 & 2 & 3 & 5 & 3 & 15 & 3 & 157 \\
\hline 4 & 1 & 4 & 3233 & 4 & 120 & 4 & 73 & 4 & 8 & 4 & 1 & 4 & 9 & 4 & 114 \\
\hline 5 & 0 & 5 & 3892 & 5 & 143 & 5 & 55 & 5 & 3 & 5 & 11 & 5 & 19 & 5 & 109 \\
\hline 6 & 3 & 6 & 3697 & 6 & 146 & 6 & 75 & 6 & 2 & 6 & 6 & 6 & 14 & 6 & 122 \\
\hline 7 & 0 & 7 & 3151 & 7 & 102 & 7 & 66 & 7 & 3 & 7 & 9 & 7 & 25 & 7 & 133 \\
\hline 8 & 0 & 8 & 3387 & 8 & 46 & 8 & 90 & 8 & 6 & 8 & 5 & 8 & 21 & 8 & 120 \\
\hline 9 & 0 & 9 & 2653 & 9 & 45 & 9 & 88 & 9 & 1 & 9 & 4 & 9 & 33 & 9 & 92 \\
\hline 10 & 0 & 10 & 2985 & 10 & 20 & 10 & 56 & 10 & 14 & 10 & 3 & 10 & 46 & 10 & 121 \\
\hline 11 & 41 & 11 & 2472 & 11 & 31 & 11 & 78 & 11 & 20 & 11 & 11 & 11 & 42 & 11 & 113 \\
\hline 12 & 0 & 12 & 2022 & 12 & 26 & 12 & 102 & 12 & 1 & 12 & 7 & 12 & 35 & 12 & 61 \\
\hline 13 & 0 & 13 & 15152 & 13 & 10 & 13 & 112 & 13 & 7 & 13 & 12 & 13 & 46 & 13 & 82 \\
\hline 14 & 0 & 14 & 4050 & 14 & 30 & 14 & 99 & 14 & 6 & 14 & 58 & 14 & 55 & 14 & 99 \\
\hline 15 & 0 & 15 & 2644 & 15 & 27 & 15 & 51 & 15 & 5 & 15 & 49 & 15 & 54 & 15 & 70 \\
\hline 16 & 0 & 16 & 2008 & 16 & 29 & 16 & 50 & 16 & 9 & 16 & 45 & 16 & 20 & 16 & 66 \\
\hline 17 & 4 & 17 & 2051 & 17 & 39 & 17 & 352 & 17 & 6 & 17 & 44 & 17 & 78 & 17 & 98 \\
\hline 18 & 17 & 18 & 1893 & 18 & 58 & 18 & 31 & 18 & 10 & 18 & 36 & 18 & 82 & 18 & 67 \\
\hline 19 & 59 & 19 & 1751 & 19 & 122 & 19 & 43 & 19 & 6 & 19 & 37 & 19 & 80 & 19 & 54 \\
\hline 20 & 77 & 20 & 396 & 20 & 116 & 20 & 16 & 20 & 5 & 20 & 30 & 20 & 131 & 20 & 33 \\
\hline 21 & 93 & 21 & 892 & 21 & 82 & 21 & 14 & 21 & 2 & 21 & 27 & 21 & 84 & 21 & 40 \\
\hline 22 & 152 & 22 & 825 & 22 & 1 & 22 & 35 & 22 & 13 & 22 & 21 & 22 & 74 & 22 & 50 \\
\hline 23 & 131 & 23 & 649 & 23 & 104 & 23 & 15 & 23 & 2 & 23 & 52 & 23 & 135 & 23 & 38 \\
\hline 24 & 261 & 24 & 221 & 24 & 164 & 24 & 9 & 24 & 3 & 24 & 28 & 24 & 139 & 24 & 41 \\
\hline 25 & 462 & 25 & 517 & 25 & 102 & 25 & 12 & 25 & 11 & 25 & 21 & 25 & 160 & 25 & 23 \\
\hline 26 & 688 & 26 & 411 & 26 & 92 & 26 & 14 & 26 & 7 & 26 & 29 & 26 & 179 & 26 & 34 \\
\hline 27 & 776 & 27 & 440 & 27 & 132 & 27 & 3 & 27 & 1 & 27 & 24 & 27 & 189 & 27 & 32 \\
\hline 28 & 1772 & 28 & 329 & 28 & 120 & 28 & 6 & 28 & 3 & 28 & 18 & 28 & 217 & 28 & 30 \\
\hline 29 & 1462 & 29 & 430 & 29 & 128 & 29 & 22 & 29 & 0 & 29 & 14 & 29 & 212 & 29 & 22 \\
\hline 30 & 1741 & & & 30 & 114 & 30 & 4 & 30 & 18 & 30 & 23 & 30 & 223 & 30 & 28 \\
\hline 31 & 1984 & & & 31 & 90 & & & 31 & 5 & & & 31 & 276 & 31 & 32 \\
\hline
\end{tabular}

Fonte: OMS (2020). 
Com esses números, foi possível encontrar $\circ X^{2}$ calculado para comparar as frequências relativas observadas do primeiro dígito desses números com as frequências relativas esperadas, de acordo com a Lei de Benford, como indica a Tabela 4.

Tabela 4 - Teste de comparação de frequências para o primeiro dígito dos números de casos confirmados de COVID-19 na China.

\begin{tabular}{cccccccc}
\hline Dígito & $\mathbf{o}_{\boldsymbol{j}}$ & $\begin{array}{c}\text { Frequência } \\
\text { relativa } \\
\text { observada }\end{array}$ & $\mathbf{e}_{\boldsymbol{j}}$ & $\begin{array}{c}\text { Frequência } \\
\text { relativa } \\
\text { esperada }\end{array}$ & $\left.\mathbf{( o}_{\boldsymbol{j}}-\mathbf{e}_{j}\right)$ & $\left(\mathbf{o}_{j}-\mathbf{e}_{j}\right)^{\mathbf{2}}$ & $\frac{\left(\mathbf{o}_{j}-\mathbf{e}_{j}\right)^{\mathbf{2}}}{\mathbf{e}_{j}}$ \\
\hline $\mathbf{1}$ & 69 & $29,9 \%$ & 69,531 & $30,1 \%$ & $-0,531$ & 0,282 & 0,004 \\
$\mathbf{2}$ & 40 & $17,3 \%$ & 40,656 & $17,6 \%$ & $-0,656$ & 0,430 & 0,011 \\
$\mathbf{3}$ & 32 & $13,9 \%$ & 28,875 & $12,5 \%$ & 3,125 & 9,766 & 0,338 \\
$\mathbf{4}$ & 21 & $9,1 \%$ & 22,407 & $9,7 \%$ & $-1,407$ & 1,980 & 0,088 \\
$\mathbf{5}$ & 20 & $8,7 \%$ & 18,249 & $7,9 \%$ & 1,751 & 3,066 & 0,168 \\
$\mathbf{6}$ & 13 & $5,6 \%$ & 15,477 & $6,7 \%$ & $-2,477$ & 6,136 & 0,396 \\
$\mathbf{7}$ & 12 & $5,2 \%$ & 13,398 & $5,8 \%$ & $-1,398$ & 1,954 & 0,146 \\
$\mathbf{8}$ & 10 & $4,3 \%$ & 11,781 & $5,1 \%$ & $-1,781$ & 3,172 & 0,269 \\
$\mathbf{9}$ & 14 & $6,1 \%$ & 10,626 & $4,6 \%$ & 3,374 & 11,384 & 1,071 \\
Total & $\mathbf{2 3 1}$ & & & & & $\boldsymbol{X}^{\mathbf{2}}$ calculado $=$ & 2,492 \\
\hline
\end{tabular}

Fonte: Elaboração do autor (2020).

Como o $X^{2}$ calculado é inferior ao $X^{2}$ crítico $(15,507)$, aceitamos a hipótese que o número de casos confirmados de COVID-19 na China segue a Lei de Benford. A Figura 2 ilustra as frequências relativas observadas e esperadas, permitindo visualmente observar como a medida de discrepância entre as frequências é pequena.

Figura 2 - Gráfico das frequências relativas observadas e esperadas no caso da China.

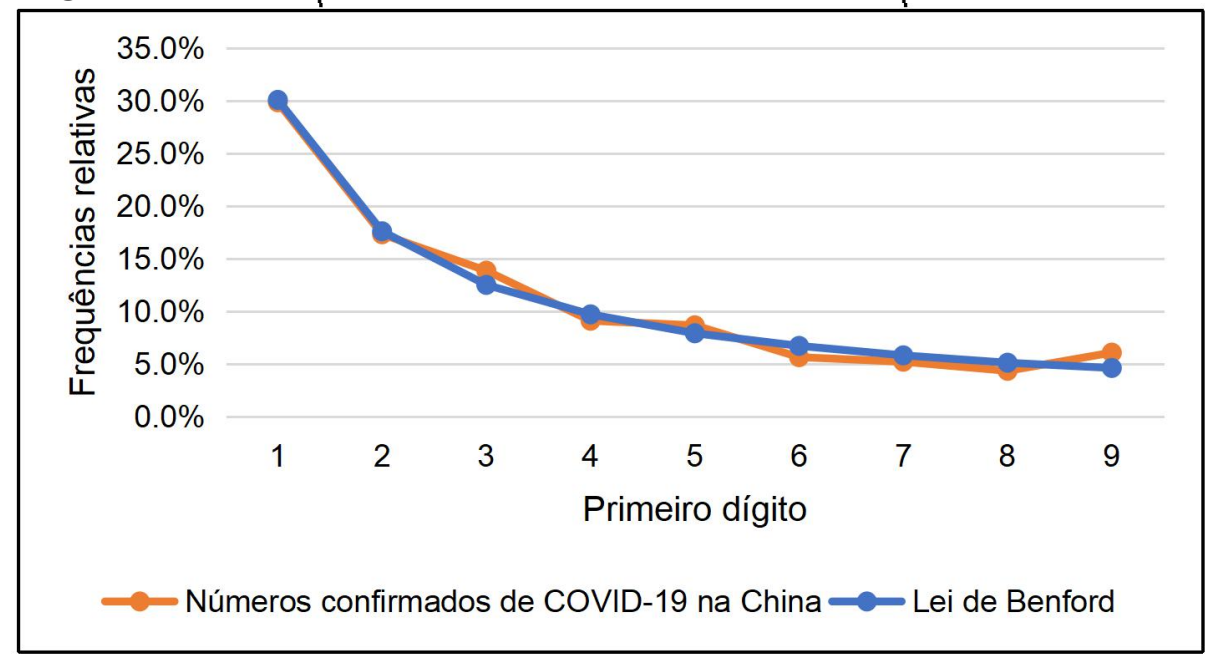

Fonte: Elaboração do autor (2020).

\subsection{Itália}

De acordo com a OMS, na Itália, de 29 de janeiro a 31 de agosto de 2020, houve 268.366 casos confirmados de COVID-19. Não consideramos o número do dia 20 de junho, pois foi computado erroneamente com um número negativo (-148). Apresentamos esses números organizados pelos dias do mês na Tabela 5 . 
Tabela 5 - Números de casos diários confirmados de COVID-19 na Itália.

\begin{tabular}{|c|c|c|c|c|c|c|c|c|c|c|c|c|c|c|c|}
\hline \multicolumn{2}{|c|}{ Janeiro } & \multicolumn{2}{|c|}{ Fevereiro } & \multicolumn{2}{|c|}{ Março } & \multicolumn{2}{|c|}{ Abril } & \multicolumn{2}{|c|}{ Maio } & \multicolumn{2}{|c|}{ Junho } & \multicolumn{2}{|c|}{ Julho } & \multicolumn{2}{|c|}{ Agosto } \\
\hline Dia & $n .^{\circ}$ & Dia & $n^{\circ}$ & Dia & $n^{\circ}$ & Dia & $n .^{\circ}$ & Dia & $n^{\circ}$ & Dia & $n .^{\circ}$ & Dia & $n .^{\circ}$ & Dia & $n^{\circ}$ \\
\hline 1 & - & 1 & 0 & 1 & 561 & 1 & 4053 & 1 & 1872 & 1 & 355 & 1 & 142 & 1 & 379 \\
\hline 2 & - & 2 & 0 & 2 & 347 & 2 & 4782 & 2 & 1965 & 2 & 178 & 2 & 182 & 2 & 295 \\
\hline 3 & - & 3 & 0 & 3 & 466 & 3 & 4668 & 3 & 1900 & 3 & 318 & 3 & 201 & 3 & 238 \\
\hline 4 & - & 4 & 0 & 4 & 587 & 4 & 4585 & 4 & 1389 & 4 & 321 & 4 & 223 & 4 & 159 \\
\hline 5 & - & 5 & 0 & 5 & 769 & 5 & 4805 & 5 & 1221 & 5 & 177 & 5 & 235 & 5 & 190 \\
\hline 6 & - & 6 & 0 & 6 & 778 & 6 & 4316 & 6 & 1075 & 6 & 518 & 6 & 192 & 6 & 384 \\
\hline 7 & - & 7 & 1 & 7 & 1247 & 7 & 3599 & 7 & 1444 & 7 & 270 & 7 & 208 & 7 & 401 \\
\hline 8 & - & 8 & 0 & 8 & 1492 & 8 & 3039 & 8 & 1401 & 8 & 197 & 8 & 137 & 8 & 552 \\
\hline 9 & - & 9 & 0 & 9 & 1797 & 9 & 3836 & 9 & 1327 & 9 & 280 & 9 & 193 & 9 & 347 \\
\hline 10 & - & 10 & 0 & 10 & 977 & 10 & 4204 & 10 & 1083 & 10 & 283 & 10 & 214 & 10 & 463 \\
\hline 11 & - & 11 & 0 & 11 & 2313 & 11 & 3951 & 11 & 802 & 11 & 202 & 11 & 276 & 11 & 259 \\
\hline 12 & - & 12 & 0 & 12 & 2651 & 12 & 4694 & 12 & 744 & 12 & 379 & 12 & 188 & 12 & 412 \\
\hline 13 & - & 13 & 0 & 13 & 2547 & 13 & 4092 & 13 & 1402 & 13 & 163 & 13 & 234 & 13 & 476 \\
\hline 14 & - & 14 & 0 & 14 & 3497 & 14 & 3153 & 14 & 888 & 14 & 346 & 14 & 169 & 14 & 522 \\
\hline 15 & - & 15 & 0 & 15 & 3590 & 15 & 2972 & 15 & 992 & 15 & 338 & 15 & 114 & 15 & 574 \\
\hline 16 & - & 16 & 0 & 16 & 3233 & 16 & 2667 & 16 & 789 & 16 & 301 & 16 & 162 & 16 & 629 \\
\hline 17 & - & 17 & 0 & 17 & 3526 & 17 & 3786 & 17 & 875 & 17 & 210 & 17 & 230 & 17 & 477 \\
\hline 18 & - & 18 & 0 & 18 & 4207 & 18 & 3493 & 18 & 675 & 18 & 328 & 18 & 231 & 18 & 320 \\
\hline 19 & - & 19 & 0 & 19 & 5318 & 19 & 3491 & 19 & 451 & 19 & 331 & 19 & 249 & 19 & 401 \\
\hline 20 & - & 20 & 0 & 20 & 5986 & 20 & 3047 & 20 & 813 & 20 & - & 20 & 218 & 20 & 642 \\
\hline 21 & - & 21 & 1 & 21 & 6557 & 21 & 2256 & 21 & 665 & 21 & 264 & 21 & 190 & 21 & 840 \\
\hline 22 & - & 22 & 7 & 22 & 0 & 22 & 2729 & 22 & 642 & 22 & 224 & 22 & 128 & 22 & 947 \\
\hline 23 & - & 23 & 113 & 23 & 5560 & 23 & 3370 & 23 & 652 & 23 & 221 & 23 & 280 & 23 & 1071 \\
\hline 24 & - & 24 & 105 & 24 & 4789 & 24 & 2646 & 24 & 669 & 24 & 113 & 24 & 306 & 24 & 1209 \\
\hline 25 & - & 25 & 93 & 25 & 5249 & 25 & 3021 & 25 & 531 & 25 & 577 & 25 & 252 & 25 & 953 \\
\hline 26 & - & 26 & 78 & 26 & 5210 & 26 & 2357 & 26 & 300 & 26 & 296 & 26 & 274 & 26 & 876 \\
\hline 27 & - & 27 & 250 & 27 & 6153 & 27 & 2324 & 27 & 397 & 27 & 255 & 27 & 254 & 27 & 1366 \\
\hline 28 & - & 28 & 238 & 28 & 5959 & 28 & 1739 & 28 & 584 & 28 & 175 & 28 & 168 & 28 & 1409 \\
\hline 29 & 6 & 29 & 240 & 29 & 5974 & 29 & 2091 & 29 & 593 & 29 & 174 & 29 & 202 & 29 & 1460 \\
\hline 30 & 0 & & & 30 & 5217 & 30 & 2086 & 30 & 516 & 30 & 126 & 30 & 288 & 30 & 1444 \\
\hline 31 & 0 & & & 31 & 4050 & & & 31 & 416 & & & 31 & 382 & 31 & 1365 \\
\hline
\end{tabular}

Fonte: Fonte: OMS (2020).

Com os números diários de casos confirmados de COVID-19 na Itália tabelados, foi possível encontrar o $\mathrm{X}^{2}$ calculado, conforme demonstra a Tabela 6 . 
Tabela 6 - Teste de comparação de frequências para o primeiro dígito dos números de casos confirmados de COVID-19 na Itália.

\begin{tabular}{cccccccc}
\hline Dígito & $\mathbf{o}_{j}$ & $\begin{array}{c}\text { Frequência } \\
\text { relativa } \\
\text { observada }\end{array}$ & $\mathbf{e}_{j}$ & $\begin{array}{c}\text { Frequência } \\
\text { relativa } \\
\text { esperada }\end{array}$ & $\left.\mathbf{( o}_{j}-\mathbf{e}_{j}\right)$ & $\left(\mathbf{o}_{j}-\mathbf{e}_{j}\right)^{\mathbf{2}}$ & $\frac{\left(\mathbf{o}_{j}-\mathbf{e}_{j}\right)^{\mathbf{2}}}{\mathbf{e}_{j}}$ \\
\hline $\mathbf{1}$ & 48 & $24,9 \%$ & 58,093 & $30,1 \%$ & $-10,093$ & 101,869 & 1,754 \\
$\mathbf{2}$ & 45 & $23,3 \%$ & 33,968 & $17,6 \%$ & 11,032 & 121,705 & 3,583 \\
$\mathbf{3}$ & 33 & $17,1 \%$ & 24,125 & $12,5 \%$ & 8,875 & 78,766 & 3,265 \\
$\mathbf{4}$ & 21 & $10,9 \%$ & 18,721 & $9,7 \%$ & 2,279 & 5,194 & 0,277 \\
$\mathbf{5}$ & 19 & $9,8 \%$ & 15,247 & $7,9 \%$ & 3,753 & 14,085 & 0,924 \\
$\mathbf{6}$ & 10 & $5,2 \%$ & 12,931 & $6,7 \%$ & $-2,931$ & 8,591 & 0,664 \\
$\mathbf{7}$ & 6 & $3,1 \%$ & 11,194 & $5,8 \%$ & $-5,194$ & 26,978 & 2,410 \\
$\mathbf{8}$ & 6 & $3,1 \%$ & 9,843 & $5,1 \%$ & $-3,843$ & 14,769 & 1,500 \\
$\mathbf{9}$ & 5 & $2,6 \%$ & 8,878 & $4,6 \%$ & $-3,878$ & 15,039 & 1,694 \\
Total & $\mathbf{1 9 3}$ & & & & & $\boldsymbol{X}^{\mathbf{2}}$ calculado $=$ & 16,071 \\
\hline
\end{tabular}

Fonte: Elaboração do autor (2020).

Para os números de casos confirmados de COVID-19 na Itália, o $X^{2}$ calculado foi maior que o $X^{2}$ crítico $(15,507)$, indicando, em um nível de significância de $5 \%$, a rejeição da hipótese $H_{0}$. A Figura 3 mostra o comportamento das frequências relativas observadas e esperadas, que confirma essa rejeição.

Figura 3 - Gráfico das frequências relativas observadas e esperadas no caso da Itália.

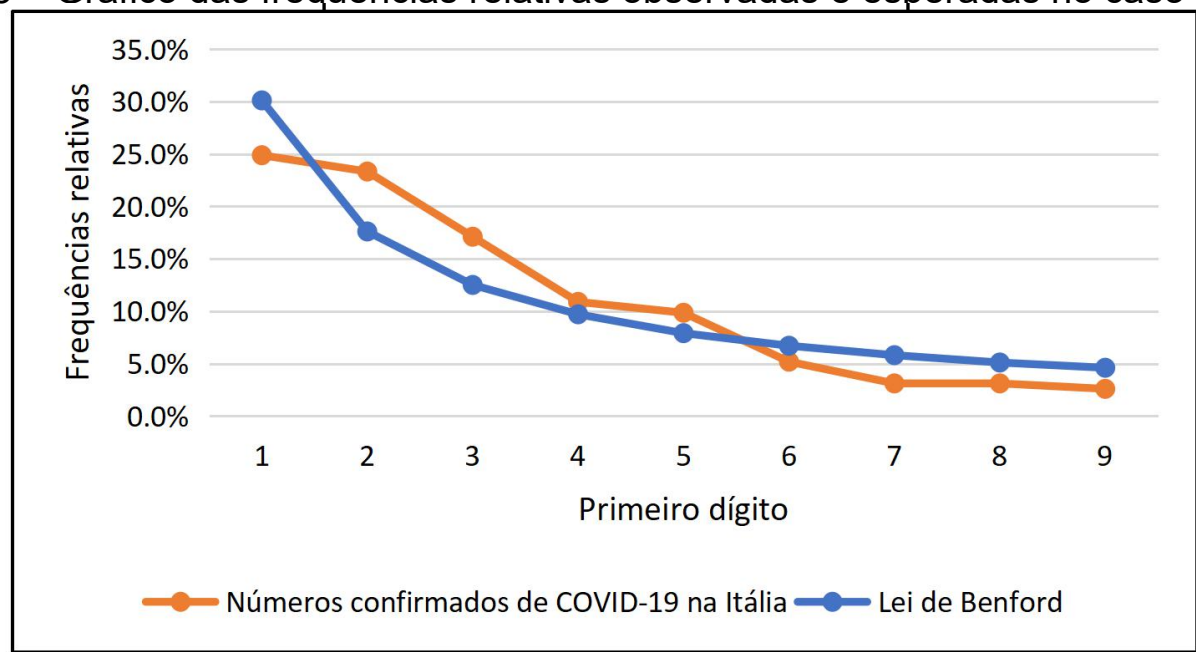

Fonte: Elaboração do autor (2020).

\subsection{Nova Zelândia}

Diferentemente da China e da Itália, que computaram os primeiros casos confirmados de COVID-19 em janeiro, na Nova Zelândia, de acordo com a OMS, de 28 de fevereiro a 31 de agosto, houve 1.387 casos confirmados de COVID-19, conforme apresentado na Tabela 7.

Tabela 7 - Números de casos diários confirmados de COVID-19 na Nova Zelândia.

\begin{tabular}{cccccccccccccc}
\hline \multicolumn{2}{c}{ Fevereiro } & \multicolumn{2}{c}{ Março } & \multicolumn{2}{c}{ Abril } & \multicolumn{2}{c}{ Maio } & \multicolumn{2}{c}{ Junho } & \multicolumn{2}{c}{ Julho } & \multicolumn{2}{c}{ Agosto } \\
Dia & n. $^{\circ}$ & Dia & n. $^{\circ}$ & Dia & n. $^{\circ}$ & Dia & n. $^{\circ}$ & Dia & n. $^{\circ}$ & Dia & n. & Dia & n. $^{\circ}$ \\
\hline $\mathbf{1}$ & - & $\mathbf{1}$ & 0 & $\mathbf{1}$ & 47 & $\mathbf{1}$ & 3 & $\mathbf{1}$ & 0 & $\mathbf{1}$ & 0 & $\mathbf{1}$ & 2 \\
$\mathbf{2}$ & - & $\mathbf{2}$ & 0 & $\mathbf{2}$ & 76 & $\mathbf{2}$ & 2 & $\mathbf{2}$ & 0 & $\mathbf{2}$ & 2 & $\mathbf{2}$ & 3 \\
$\mathbf{3}$ & - & $\mathbf{3}$ & 1 & $\mathbf{3}$ & 51 & $\mathbf{3}$ & 2 & $\mathbf{3}$ & 0 & $\mathbf{3}$ & 0 & $\mathbf{3}$ & 2
\end{tabular}




\begin{tabular}{cccccccccccccc}
$\mathbf{4}$ & - & $\mathbf{4}$ & 0 & $\mathbf{4}$ & 50 & $\mathbf{4}$ & 1 & $\mathbf{4}$ & 0 & $\mathbf{4}$ & 0 & $\mathbf{4}$ & 0 \\
$\mathbf{5}$ & - & $\mathbf{5}$ & 0 & $\mathbf{5}$ & 48 & $\mathbf{5}$ & 0 & $\mathbf{5}$ & 0 & $\mathbf{5}$ & 3 & $\mathbf{5}$ & 2 \\
$\mathbf{6}$ & - & $\mathbf{6}$ & 2 & $\mathbf{6}$ & 39 & $\mathbf{6}$ & 1 & $\mathbf{6}$ & 0 & $\mathbf{6}$ & 1 & $\mathbf{6}$ & 0 \\
$\mathbf{7}$ & - & $\mathbf{7}$ & 1 & $\mathbf{7}$ & 32 & $\mathbf{7}$ & 1 & $\mathbf{7}$ & 0 & $\mathbf{7}$ & 2 & $\mathbf{7}$ & 0 \\
$\mathbf{8}$ & - & $\mathbf{8}$ & 0 & $\mathbf{8}$ & 26 & $\mathbf{8}$ & 2 & $\mathbf{8}$ & 0 & $\mathbf{8}$ & 1 & $\mathbf{8}$ & 0 \\
$\mathbf{9}$ & - & $\mathbf{9}$ & 0 & $\mathbf{9}$ & 23 & $\mathbf{9}$ & 1 & $\mathbf{9}$ & 0 & $\mathbf{9}$ & 3 & $\mathbf{9}$ & 0 \\
$\mathbf{1 0}$ & - & $\mathbf{1 0}$ & 0 & $\mathbf{1 0}$ & 23 & $\mathbf{1 0}$ & 2 & $\mathbf{1 0}$ & 0 & $\mathbf{1 0}$ & 2 & $\mathbf{1 0}$ & 0 \\
$\mathbf{1 1}$ & - & $\mathbf{1 1}$ & 0 & $\mathbf{1 1}$ & 20 & $\mathbf{1 1}$ & 3 & $\mathbf{1 1}$ & 0 & $\mathbf{1 1}$ & 1 & $\mathbf{1 1}$ & 1 \\
$\mathbf{1 2}$ & - & $\mathbf{1 2}$ & 0 & $\mathbf{1 2}$ & 14 & $\mathbf{1 2}$ & 0 & $\mathbf{1 2}$ & 0 & $\mathbf{1 2}$ & 1 & $\mathbf{1 2}$ & 4 \\
$\mathbf{1 3}$ & - & $\mathbf{1 3}$ & 0 & $\mathbf{1 3}$ & 15 & $\mathbf{1 3}$ & 0 & $\mathbf{1 3}$ & 0 & $\mathbf{1 3}$ & 0 & $\mathbf{1 3}$ & 14 \\
$\mathbf{1 4}$ & - & $\mathbf{1 4}$ & 1 & $\mathbf{1 4}$ & 8 & $\mathbf{1 4}$ & 0 & $\mathbf{1 4}$ & 0 & $\mathbf{1 4}$ & 1 & $\mathbf{1 4}$ & 13 \\
$\mathbf{1 5}$ & - & $\mathbf{1 5}$ & 0 & $\mathbf{1 5}$ & 6 & $\mathbf{1 5}$ & 1 & $\mathbf{1 5}$ & 0 & $\mathbf{1 5}$ & 2 & $\mathbf{1 5}$ & 7 \\
$\mathbf{1 6}$ & - & $\mathbf{1 6}$ & 0 & $\mathbf{1 6}$ & 6 & $\mathbf{1 6}$ & 0 & $\mathbf{1 6}$ & 2 & $\mathbf{1 6}$ & 1 & $\mathbf{1 6}$ & 13 \\
$\mathbf{1 7}$ & - & $\mathbf{1 7}$ & 5 & $\mathbf{1 7}$ & 2 & $\mathbf{1 7}$ & 1 & $\mathbf{1 7}$ & 0 & $\mathbf{1 7}$ & 1 & $\mathbf{1 7}$ & 9 \\
$\mathbf{1 8}$ & - & $\mathbf{1 8}$ & 9 & $\mathbf{1 8}$ & 8 & $\mathbf{1 8}$ & 0 & $\mathbf{1 8}$ & 1 & $\mathbf{1 8}$ & 1 & $\mathbf{1 8}$ & 13 \\
$\mathbf{1 9}$ & - & $\mathbf{1 9}$ & 15 & $\mathbf{1 9}$ & 4 & $\mathbf{1 9}$ & 4 & $\mathbf{1 9}$ & 0 & $\mathbf{1 9}$ & 3 & $\mathbf{1 9}$ & 6 \\
$\mathbf{2 0}$ & - & $\mathbf{2 0}$ & 14 & $\mathbf{2 0}$ & 7 & $\mathbf{2 0}$ & 0 & $\mathbf{2 0}$ & 2 & $\mathbf{2 0}$ & 1 & $\mathbf{2 0}$ & 5 \\
$\mathbf{2 1}$ & - & $\mathbf{2 1}$ & 13 & $\mathbf{2 1}$ & 2 & $\mathbf{2 1}$ & 0 & $\mathbf{2 1}$ & 0 & $\mathbf{2 1}$ & 1 & $\mathbf{2 1}$ & 11 \\
$\mathbf{2 2}$ & - & $\mathbf{2 2}$ & 0 & $\mathbf{2 2}$ & 5 & $\mathbf{2 2}$ & 1 & $\mathbf{2 2}$ & 4 & $\mathbf{2 2}$ & 0 & $\mathbf{2 2}$ & 6 \\
$\mathbf{2 3}$ & - & $\mathbf{2 3}$ & 36 & $\mathbf{2 3}$ & 0 & $\mathbf{2 3}$ & 0 & $\mathbf{2 3}$ & 2 & $\mathbf{2 3}$ & 0 & $\mathbf{2 3}$ & 3 \\
$\mathbf{2 4}$ & - & $\mathbf{2 4}$ & 40 & $\mathbf{2 4}$ & 2 & $\mathbf{2 4}$ & 0 & $\mathbf{2 4}$ & 1 & $\mathbf{2 4}$ & 1 & $\mathbf{2 4}$ & 8 \\
$\mathbf{2 5}$ & - & $\mathbf{2 5}$ & 47 & $\mathbf{2 5}$ & 3 & $\mathbf{2 5}$ & 0 & $\mathbf{2 5}$ & 3 & $\mathbf{2 5}$ & 0 & $\mathbf{2 5}$ & 7 \\
$\mathbf{2 6}$ & - & $\mathbf{2 6}$ & 73 & $\mathbf{2 6}$ & 4 & $\mathbf{2 6}$ & 0 & $\mathbf{2 6}$ & 1 & $\mathbf{2 6}$ & 0 & $\mathbf{2 6}$ & 5 \\
$\mathbf{2 7}$ & - & $\mathbf{2 7}$ & 76 & $\mathbf{2 7}$ & 1 & $\mathbf{2 7}$ & 0 & $\mathbf{2 7}$ & 2 & $\mathbf{2 7}$ & 0 & $\mathbf{2 7}$ & 7 \\
$\mathbf{2 8}$ & 5 & $\mathbf{2 8}$ & 78 & $\mathbf{2 8}$ & 2 & $\mathbf{2 8}$ & 0 & $\mathbf{2 8}$ & 4 & $\mathbf{2 8}$ & 1 & $\mathbf{2 8}$ & 12 \\
$\mathbf{2 9}$ & 0 & $\mathbf{2 9}$ & 60 & $\mathbf{2 9}$ & 2 & $\mathbf{2 9}$ & 0 & $\mathbf{2 9}$ & 2 & $\mathbf{2 9}$ & 1 & $\mathbf{2 9}$ & 13 \\
& & $\mathbf{3 0}$ & 76 & $\mathbf{3 0}$ & 3 & $\mathbf{3 0}$ & 0 & $\mathbf{3 0}$ & 0 & $\mathbf{3 0}$ & 2 & $\mathbf{3 0}$ & 2 \\
& & $\mathbf{3 1}$ & 48 & & & $\mathbf{3 1}$ & 0 & & & $\mathbf{3 1}$ & 0 & $\mathbf{3 1}$ & 9 \\
\hline
\end{tabular}

Fonte: OMS (2020).

Com os números de casos confirmados de COVID-19 na Nova Zelândia, foi possível encontrar o $X^{2}$ calculado, conforme apresentado na Tabela 8.

Tabela 8 - Teste de comparação de frequências para o primeiro dígito dos números de casos confirmados de COVID-19 na Nova Zelândia.

\begin{tabular}{|c|c|c|c|c|c|c|c|}
\hline Dígito & $o_{j}$ & $\begin{array}{c}\text { Frequência } \\
\text { relativa } \\
\text { observada }\end{array}$ & $\mathbf{e}_{j}$ & $\begin{array}{c}\text { Frequência } \\
\text { relativa } \\
\text { esperada }\end{array}$ & $\left(o_{j}-e_{j}\right)$ & $\left(o_{j}-e_{j}\right)^{2}$ & $\frac{\left(o_{j}-e_{j}\right)^{2}}{e_{j}}$ \\
\hline 1 & 40 & $33,6 \%$ & 35,819 & $30,1 \%$ & 4,181 & 17,481 & 0,488 \\
\hline 2 & 28 & $23,5 \%$ & 20,944 & $17,6 \%$ & 7,056 & 49,787 & 2,377 \\
\hline 3 & 13 & $10,9 \%$ & 14,875 & $12,5 \%$ & $-1,875$ & 3,516 & 0,236 \\
\hline 4 & 11 & $9,2 \%$ & 11,543 & $9,7 \%$ & $-0,543$ & 0,295 & 0,026 \\
\hline 5 & 7 & $5,9 \%$ & 9,401 & $7,9 \%$ & $-2,401$ & 5,765 & 0,613 \\
\hline 6 & 5 & $4,2 \%$ & 7,973 & $6,7 \%$ & $-2,973$ & 8,839 & 1,109 \\
\hline 7 & 9 & $7,6 \%$ & 6,902 & $5,8 \%$ & 2,098 & 4,402 & 0,638 \\
\hline 8 & 3 & $2,5 \%$ & 6,069 & $5,1 \%$ & $-3,069$ & 9,419 & 1,552 \\
\hline 9 & 3 & $2,5 \%$ & 5,474 & $4,6 \%$ & $-2,474$ & 6,121 & 1,118 \\
\hline Total & 119 & & & & & $X^{2}$ calculado $=$ & 8,157 \\
\hline
\end{tabular}

Fonte: Elaboração do autor (2020). 
Para os números de casos confirmados de COVID-19 na Nova Zelândia, o $X^{2}$ calculado foi menor que o valor de 15,507 do $X^{2}$ crítico, indicando, em um nível de significância de $5 \%$, o aceite da hipótese $H_{0}$. Na Figura 4, é possível observar o comportamento das frequências relativas observadas e esperadas para os dígitos considerados pela Lei de Benford.

Figura 4 - Gráfico das frequências relativas observadas e esperadas no caso da Nova Zelândia.

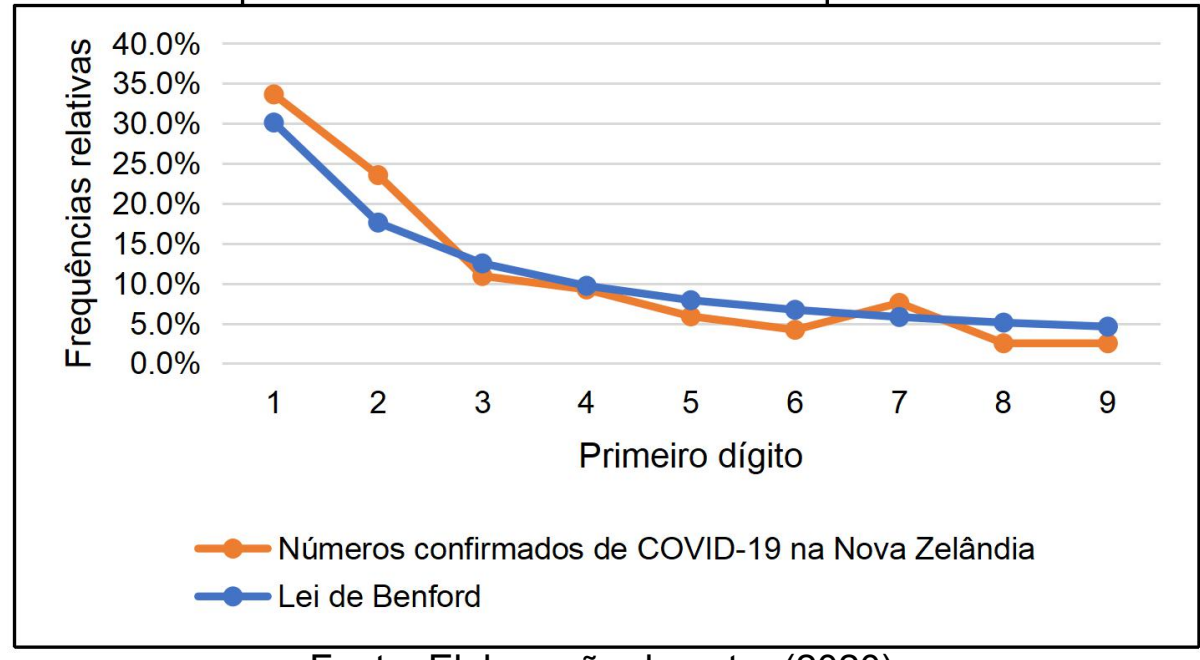

Fonte: Elaboração do autor (2020).

\subsection{Brasil}

Os números de casos confirmados de COVID-19 no Brasil, assim como na Nova Zelândia, também começaram a ser computados no final da segunda quinzena do mês de fevereiro. $E$, de acordo com a OMS, de 26 de fevereiro a 31 de agosto, houve 3.846 .153 casos confirmados de COVID-19, como apresentado na Tabela 9.

Tabela 9 - Números de casos diários confirmados de COVID-19 no Brasil.

\begin{tabular}{|c|c|c|c|c|c|c|c|c|c|c|c|c|c|}
\hline \multicolumn{2}{|c|}{ Fevereiro } & \multicolumn{2}{|c|}{ Marco } & \multicolumn{2}{|c|}{ Abril } & \multicolumn{2}{|c|}{ Maio } & \multicolumn{2}{|c|}{ Junho } & \multicolumn{2}{|c|}{ Julho } & \multicolumn{2}{|c|}{ Agosto } \\
\hline Dia & $n .^{\circ}$ & Dia & n. & Dia & $n .^{\circ}$ & Dia & $n .^{\circ}$ & Dia & $n .^{\circ}$ & Dia & $n .^{\circ}$ & Dia & $n .^{\circ}$ \\
\hline 1 & - & 1 & 1 & 1 & 323 & 1 & 6276 & 1 & 33274 & 1 & 24052 & 1 & 57837 \\
\hline 2 & - & 2 & 0 & 2 & 1138 & 2 & 7218 & 2 & 16409 & 2 & 33846 & 2 & 52383 \\
\hline 3 & - & 3 & 0 & 3 & 1119 & 3 & 6209 & 3 & 11598 & 3 & 46712 & 3 & 45392 \\
\hline 4 & - & 4 & 1 & 4 & 1074 & 4 & 4970 & 4 & 28936 & 4 & 48105 & 4 & 25800 \\
\hline 5 & - & 5 & 4 & 5 & 1146 & 5 & 4588 & 5 & 28633 & 5 & 42223 & 5 & 16641 \\
\hline 6 & - & 6 & 1 & 6 & 1222 & 6 & 6633 & 6 & 30925 & 6 & 37923 & 6 & 51603 \\
\hline 7 & - & 7 & 11 & 7 & 852 & 7 & 6935 & 7 & 30830 & 7 & 26051 & 7 & 57152 \\
\hline 8 & - & 8 & 0 & 8 & 926 & 8 & 10503 & 8 & 27075 & 8 & 20229 & 8 & 53139 \\
\hline 9 & - & 9 & 6 & 9 & 1661 & 9 & 9888 & 9 & 18912 & 9 & 45305 & 9 & 50230 \\
\hline 10 & - & 10 & 0 & 10 & 2210 & 10 & 10222 & 10 & 15654 & 10 & 44571 & 10 & 49970 \\
\hline 11 & - & 11 & 9 & 11 & 1930 & 11 & 10611 & 11 & 32091 & 11 & 42619 & 11 & 23010 \\
\hline 12 & - & 12 & 18 & 12 & 1781 & 12 & 6760 & 12 & 32913 & 12 & 45048 & 12 & 22048 \\
\hline 13 & - & 13 & 46 & 13 & 1089 & 13 & 5632 & 13 & 30412 & 13 & 39023 & 13 & 52160 \\
\hline 14 & - & 14 & 23 & 14 & 1442 & 14 & 9258 & 14 & 25982 & 14 & 24831 & 14 & 55155 \\
\hline 15 & - & 15 & 0 & 15 & 1261 & 15 & 11385 & 15 & 21704 & 15 & 20286 & 15 & 60091 \\
\hline 16 & - & 16 & 79 & 16 & 1832 & 16 & 13944 & 16 & 17110 & 16 & 41857 & 16 & 50644 \\
\hline 17 & - & 17 & 34 & 17 & 3058 & 17 & 15305 & 17 & 20647 & 17 & 39924 & 17 & 41576 \\
\hline 18 & - & 18 & 57 & 18 & 2105 & 18 & 14919 & 18 & 34918 & 18 & 45403 & 18 & 23101 \\
\hline
\end{tabular}




\begin{tabular}{cccccccccccccc}
$\mathbf{1 9}$ & - & $\mathbf{1 9}$ & 133 & $\mathbf{1 9}$ & 3257 & $\mathbf{1 9}$ & 7938 & $\mathbf{1 9}$ & 32188 & $\mathbf{1 9}$ & 34177 & $\mathbf{1 9}$ & 19373 \\
$\mathbf{2 0}$ & - & $\mathbf{2 0}$ & 193 & $\mathbf{2 0}$ & 2917 & $\mathbf{2 0}$ & 13140 & $\mathbf{2 0}$ & 22765 & $\mathbf{2 0}$ & 28532 & $\mathbf{2 0}$ & 47784 \\
$\mathbf{2 1}$ & - & $\mathbf{2 1}$ & 283 & $\mathbf{2 1}$ & 2055 & $\mathbf{2 1}$ & 17408 & $\mathbf{2 1}$ & 54771 & $\mathbf{2 1}$ & 23529 & $\mathbf{2 1}$ & 49298 \\
$\mathbf{2 2}$ & - & $\mathbf{2 2}$ & 0 & $\mathbf{2 2}$ & 1927 & $\mathbf{2 2}$ & 19951 & $\mathbf{2 2}$ & 34666 & $\mathbf{2 2}$ & 20257 & $\mathbf{2 2}$ & 45323 \\
$\mathbf{2 3}$ & - & $\mathbf{2 3}$ & 0 & $\mathbf{2 3}$ & 2498 & $\mathbf{2 3}$ & 18508 & $\mathbf{2 3}$ & 17459 & $\mathbf{2 3}$ & 41008 & $\mathbf{2 3}$ & 30355 \\
$\mathbf{2 4}$ & - & $\mathbf{2 4}$ & 1297 & $\mathbf{2 4}$ & 2678 & $\mathbf{2 4}$ & 20803 & $\mathbf{2 4}$ & 21432 & $\mathbf{2 4}$ & 67860 & $\mathbf{2 4}$ & 50032 \\
$\mathbf{2 5}$ & - & $\mathbf{2 5}$ & 0 & $\mathbf{2 5}$ & 3735 & $\mathbf{2 5}$ & 16508 & $\mathbf{2 5}$ & 39436 & $\mathbf{2 5}$ & 59961 & $\mathbf{2 5}$ & 23421 \\
$\mathbf{2 6}$ & 5 & $\mathbf{2 6}$ & 232 & $\mathbf{2 6}$ & 3503 & $\mathbf{2 6}$ & 15813 & $\mathbf{2 6}$ & 42725 & $\mathbf{2 6}$ & 55891 & $\mathbf{2 6}$ & 17078 \\
$\mathbf{2 7}$ & 0 & $\mathbf{2 7}$ & 0 & $\mathbf{2 7}$ & 5514 & $\mathbf{2 7}$ & 11687 & $\mathbf{2 7}$ & 39483 & $\mathbf{2 7}$ & 51147 & $\mathbf{2 7}$ & 47134 \\
$\mathbf{2 8}$ & 0 & $\mathbf{2 8}$ & 482 & $\mathbf{2 8}$ & 3379 & $\mathbf{2 8}$ & 16324 & $\mathbf{2 8}$ & 46860 & $\mathbf{2 8}$ & 24578 & $\mathbf{2 8}$ & 47161 \\
$\mathbf{2 9}$ & 0 & $\mathbf{2 9}$ & 502 & $\mathbf{2 9}$ & 4613 & $\mathbf{2 9}$ & 20599 & $\mathbf{2 9}$ & 38693 & $\mathbf{2 9}$ & 23284 & $\mathbf{2 9}$ & 44235 \\
& & $\mathbf{3 0}$ & 487 & $\mathbf{3 0}$ & 5385 & $\mathbf{3 0}$ & 26417 & $\mathbf{3 0}$ & 30476 & $\mathbf{3 0}$ & 40816 & $\mathbf{3 0}$ & 43412 \\
& & $\mathbf{3 1}$ & 352 & & & $\mathbf{3 1}$ & 26928 & & & $\mathbf{3 1}$ & 69074 & $\mathbf{3 1}$ & 41350 \\
\hline
\end{tabular}

Fonte: OMS (2020).

Considerando os números de casos confirmados de COVID-19 no Brasil, encontramos o valor de $X^{2}$ calculado, como apontado na Tabela 10.

Tabela 10 - Teste de comparação de frequências para o primeiro dígito dos números de casos confirmados de COVID-19 no Brasil.

\begin{tabular}{cccccccc}
\hline Dígito & $\mathbf{o}_{\boldsymbol{j}}$ & $\begin{array}{c}\text { Frequência } \\
\text { relativa } \\
\text { observada }\end{array}$ & $\mathbf{e}_{j}$ & $\begin{array}{c}\text { Frequência } \\
\text { relativa } \\
\text { esperada }\end{array}$ & $\left.\mathbf{( o}_{j}-\mathbf{e}_{j}\right)$ & $\left(\mathbf{o}_{j}-\mathbf{e}_{j}\right)^{\mathbf{2}}$ & $\frac{\left(\mathbf{o}_{j}-\mathbf{e}_{j}\right)^{\mathbf{2}}}{\mathbf{e}_{j}}$ \\
\hline $\mathbf{1}$ & 45 & $25,6 \%$ & 52,976 & $30,1 \%$ & $-7,976$ & 63,617 & 1,201 \\
$\mathbf{2}$ & 36 & $20,5 \%$ & 30,976 & $17,6 \%$ & 5,024 & 25,241 & 0,815 \\
$\mathbf{3}$ & 27 & $15,3 \%$ & 22,000 & $12,5 \%$ & 5,000 & 25,000 & 1,136 \\
$\mathbf{4}$ & 31 & $17,6 \%$ & 17,072 & $9,7 \%$ & 13,928 & 193,989 & 11,363 \\
$\mathbf{5}$ & 20 & $11,4 \%$ & 13,904 & $7,9 \%$ & 6,096 & 37,161 & 2,673 \\
$\mathbf{6}$ & 9 & $5,1 \%$ & 11,792 & $6,7 \%$ & $-2,792$ & 7,795 & 0,661 \\
$\mathbf{7}$ & 3 & $1,7 \%$ & 10,208 & $5,8 \%$ & $-7,208$ & 51,955 & 5,090 \\
$\mathbf{8}$ & 1 & $0,6 \%$ & 8,976 & $5,1 \%$ & $-7,976$ & 63,617 & 7,087 \\
$\mathbf{9}$ & 4 & $2,3 \%$ & 8,096 & $4,6 \%$ & $-4,096$ & 16,777 & 2,072 \\
Total & $\mathbf{1 7 6}$ & & & & & $\boldsymbol{X}^{\mathbf{2}}$ calculado $=$ & 32,098 \\
\hline
\end{tabular}

Fonte: Elaboração do autor (2020).

Os números de casos confirmados de COVID-19 no Brasil geraram um $X^{2}$ calculado maior que o $X^{2}$ crítico $(15,507)$. Indicaram uma grande discrepância entre as frequências relativas observadas e as frequências relativas esperadas, fazendo com que a hipótese $H_{0}$ fosse rejeitada para esse conjunto de números, em um nível de significância de $5 \%$. Visualmente, na Figura 5 , é possivel perceber como as frequências relativas observadas não coincidem com as frequências relativas esperadas em nenhum dígito. 
Figura 5 - Gráfico das frequências relativas observadas e esperadas no caso do Brasil.

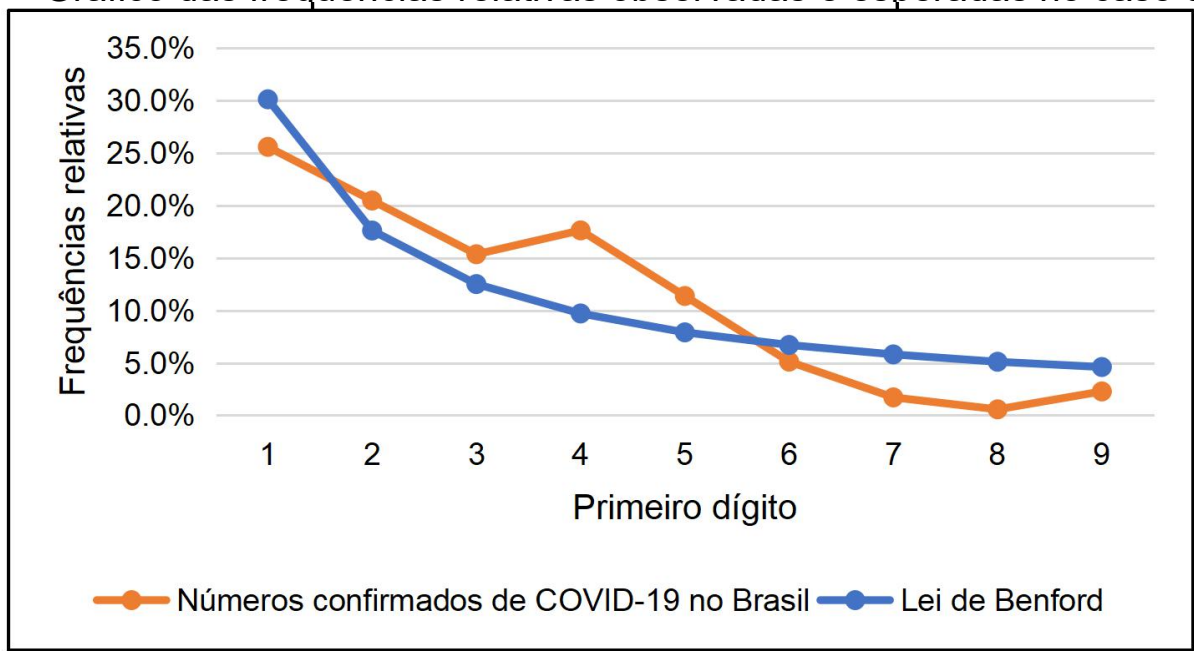

Fonte: Elaboração do autor (2020).

\subsection{Estados Unidos da América (EUA)}

Os primeiros casos confirmados de COVID-19 nos EUA começaram em janeiro. Segundo a OMS, de 20 de janeiro a 31 de agosto de 2020, houve 5.899.504 casos confirmados de COVID19 no país. Os números diários foram organizados na Tabela 11.

Tabela 11 - Números de casos diários confirmados de COVID-19 nos EUA.

\begin{tabular}{|c|c|c|c|c|c|c|c|c|c|c|c|c|c|c|c|}
\hline \multicolumn{2}{|c|}{ Janeiro } & \multicolumn{2}{|c|}{ Fevereiro } & \multicolumn{2}{|c|}{ Março } & \multicolumn{2}{|c|}{ Abril } & \multicolumn{2}{|c|}{ Maio } & \multicolumn{2}{|c|}{ Junho } & \multicolumn{2}{|c|}{ Julho } & \multicolumn{2}{|c|}{ Agosto } \\
\hline Dia & $n^{\circ}$ & Dia & $n^{\circ}$ & Dia & $n^{0}$ & Dia & $n .^{\circ}$ & Dia & $n .^{\circ}$ & Dia & $n^{\circ}$ & Dia & $n .^{\circ}$ & Dia & $n^{\circ}$ \\
\hline 1 & - & 1 & 1 & 1 & 0 & 1 & 22559 & 1 & 31379 & 1 & 23482 & 1 & 35757 & 1 & 67823 \\
\hline 2 & - & 2 & 0 & 2 & 2 & 2 & 24103 & 2 & 31774 & 2 & 26116 & 2 & 43556 & 2 & 67499 \\
\hline 3 & - & 3 & 3 & 3 & 44 & 3 & 26298 & 3 & 26753 & 3 & 14692 & 3 & 54271 & 3 & 58388 \\
\hline 4 & - & 4 & 0 & 4 & 21 & 4 & 28103 & 4 & 31839 & 4 & 24890 & 4 & 53213 & 4 & 47183 \\
\hline 5 & - & 5 & 0 & 5 & 19 & 5 & 32105 & 5 & 29266 & 5 & 14583 & 5 & 51933 & 5 & 49151 \\
\hline 6 & - & 6 & 1 & 6 & 65 & 6 & 33510 & 6 & 16200 & 6 & 20069 & 6 & 57186 & 6 & 49629 \\
\hline 7 & - & 7 & 0 & 7 & 0 & 7 & 26493 & 7 & 22267 & 7 & 28922 & 7 & 43686 & 7 & 53373 \\
\hline 8 & - & 8 & 0 & 8 & 0 & 8 & 29510 & 8 & 22119 & 8 & 28918 & 8 & 46194 & 8 & 55318 \\
\hline 9 & - & 9 & 0 & 9 & 259 & 9 & 31709 & 9 & 30204 & 9 & 17848 & 9 & 50263 & 9 & 61028 \\
\hline 10 & - & 10 & 0 & 10 & 224 & 10 & 30859 & 10 & 25870 & 10 & 17536 & 10 & 64630 & 10 & 53893 \\
\hline 11 & - & 11 & 1 & 11 & 0 & 11 & 35386 & 11 & 26642 & 11 & 17235 & 11 & 58975 & 11 & 47964 \\
\hline 12 & - & 12 & 0 & 12 & 291 & 12 & 31606 & 12 & 23767 & 12 & 20315 & 12 & 66281 & 12 & 39894 \\
\hline 13 & - & 13 & 2 & 13 & 277 & 13 & 31633 & 13 & 18044 & 13 & 21745 & 13 & 62369 & 13 & 54791 \\
\hline 14 & - & 14 & 0 & 14 & 414 & 14 & 29308 & 14 & 21424 & 14 & 22133 & 14 & 60113 & 14 & 55907 \\
\hline 15 & - & 15 & 0 & 15 & 36 & 15 & 24446 & 15 & 20840 & 15 & 25314 & 15 & 58720 & 15 & 52799 \\
\hline 16 & - & 16 & 0 & 16 & 0 & 16 & 25802 & 16 & 27090 & 16 & 21754 & 16 & 60711 & 16 & 55359 \\
\hline 17 & - & 17 & 0 & 17 & 1822 & 17 & 28711 & 17 & 22813 & 17 & 18514 & 17 & 67165 & 17 & 54375 \\
\hline 18 & - & 18 & 0 & 18 & 3551 & 18 & 32549 & 18 & 31967 & 18 & 27921 & 18 & 71484 & 18 & 41073 \\
\hline 19 & - & 19 & 0 & 19 & 3351 & 19 & 30023 & 19 & 13227 & 19 & 23139 & 19 & 74354 & 19 & 39125 \\
\hline 20 & 5 & 20 & 0 & 20 & 4777 & 20 & 28252 & 20 & 24417 & 20 & 23046 & 20 & 66963 & 20 & 37908 \\
\hline 21 & 0 & 21 & 19 & 21 & 0 & 21 & 27668 & 21 & 23310 & 21 & 36617 & 21 & 62788 & 21 & 46259 \\
\hline 22 & 0 & 22 & 1 & 22 & 0 & 22 & 25634 & 22 & 22787 & 22 & 32349 & 22 & 57276 & 22 & 43952 \\
\hline 23 & 0 & 23 & 0 & 23 & 16354 & 23 & 24019 & 23 & 20475 & 23 & 27575 & 23 & 62929 & 23 & 45960 \\
\hline 24 & 1 & 24 & 18 & 24 & 20341 & 24 & 29127 & 24 & 24151 & 24 & 26519 & 24 & 69641 & 24 & 44946 \\
\hline 25 & 0 & 25 & 0 & 25 & 0 & 25 & 30719 & 25 & 26158 & 25 & 34191 & 25 & 71714 & 25 & 37765 \\
\hline
\end{tabular}




\begin{tabular}{llllllllllllllll}
$\mathbf{2 6}$ & 3 & $\mathbf{2 6}$ & 6 & $\mathbf{2 6}$ & 16420 & $\mathbf{2 6}$ & 38509 & $\mathbf{2 6}$ & 15253 & $\mathbf{2 6}$ & 37601 & $\mathbf{2 6}$ & 74235 & $\mathbf{2 6}$ & 32883 \\
$\mathbf{2 7}$ & 0 & $\mathbf{2 7}$ & 0 & $\mathbf{2 7}$ & 0 & $\mathbf{2 7}$ & 32417 & $\mathbf{2 7}$ & 24886 & $\mathbf{2 7}$ & 40526 & $\mathbf{2 7}$ & 63968 & $\mathbf{2 7}$ & 37030 \\
$\mathbf{2 8}$ & 0 & $\mathbf{2 8}$ & 3 & $\mathbf{2 8}$ & 16894 & $\mathbf{2 8}$ & 29218 & $\mathbf{2 8}$ & 16362 & $\mathbf{2 8}$ & 44458 & $\mathbf{2 8}$ & 61498 & $\mathbf{2 8}$ & 45484 \\
$\mathbf{2 9}$ & 0 & $\mathbf{2 9}$ & 0 & $\mathbf{2 9}$ & 18093 & $\mathbf{2 9}$ & 22541 & $\mathbf{2 9}$ & 19606 & $\mathbf{2 9}$ & 44580 & $\mathbf{2 9}$ & 54022 & $\mathbf{2 9}$ & 46194 \\
$\mathbf{3 0}$ & 1 & & & $\mathbf{3 0}$ & 19332 & $\mathbf{3 0}$ & 20517 & $\mathbf{3 0}$ & 21214 & $\mathbf{3 0}$ & 41008 & $\mathbf{3 0}$ & 59629 & $\mathbf{3 0}$ & 44002 \\
$\mathbf{3 1}$ & 1 & & & $\mathbf{3 1}$ & 17987 & & & $\mathbf{3 1}$ & $\mathbf{1 7 9 6 2}$ & & & $\mathbf{3 1}$ & 65406 & $\mathbf{3 1}$ & $\mathbf{4 3 9 8 3}$ \\
\hline
\end{tabular}

Fonte: OMS (2020).

Com os números diários de casos confirmados de COVID-19 nos EUA, foi possível encontrar o $X^{2}$ calculado, como apresentado na Tabela 12.

Tabela 12 - Teste de comparação de frequências para o primeiro dígito dos números de casos confirmados de COVID-19 nos EUA.

\begin{tabular}{cccccccc}
\hline Dígito & $\mathbf{o}_{j}$ & $\begin{array}{c}\text { Frequência } \\
\text { relativa } \\
\text { observada }\end{array}$ & $\mathbf{e}_{j}$ & $\begin{array}{c}\text { Frequência } \\
\text { relativa } \\
\text { esperada }\end{array}$ & $\left(\mathbf{o}_{j}-\mathbf{e}_{j}\right)$ & $\left(\mathbf{o}_{j}-\mathbf{e}_{j}\right)^{\mathbf{2}}$ & $\frac{\left(\mathbf{o}_{j}-\mathbf{e}_{j}\right)^{\mathbf{2}}}{\mathbf{e}_{j}}$ \\
\hline $\mathbf{1}$ & 30 & $15,8 \%$ & 57,190 & $30,1 \%$ & $-27,190$ & 739,296 & 12,927 \\
$\mathbf{2}$ & 61 & $32,1 \%$ & 33,440 & $17,6 \%$ & 27,560 & 759,554 & 22,714 \\
$\mathbf{3}$ & 34 & $17,9 \%$ & 23,750 & $12,5 \%$ & 10,250 & 105,063 & 4,424 \\
$\mathbf{4}$ & 23 & $12,1 \%$ & 18,430 & $9,7 \%$ & 4,570 & 20,885 & 1,133 \\
$\mathbf{5}$ & 20 & $10,5 \%$ & 15,010 & $7,9 \%$ & 4,990 & 24,900 & 1,659 \\
$\mathbf{6}$ & 18 & $9,5 \%$ & 12,730 & $6,7 \%$ & 5,270 & 27,773 & 2,182 \\
$\mathbf{7}$ & 4 & $2,1 \%$ & 11,020 & $5,8 \%$ & $-7,020$ & 49,280 & 4,472 \\
$\mathbf{8}$ & 0 & $0,0 \%$ & 9,690 & $5,1 \%$ & $-9,690$ & 93,896 & 9,690 \\
$\mathbf{9}$ & 0 & $0,0 \%$ & 8,740 & $4,6 \%$ & $-8,740$ & 76,388 & 8,740 \\
Total & 190 & & & & & $\boldsymbol{X}^{\mathbf{2}}$ calculado $=$ & 67,940 \\
\hline
\end{tabular}

Fonte: Elaboração do autor (2020).

O $X^{2}$ calculado foi de 67,940 , enquanto o $X^{2}$ crítico é de 15,507. Dessa forma, é possível concluir que o $X^{2}$ calculado foi maior que o $X^{2}$ crítico, o que indica, em um nível de $5 \%$ de significância, que a hipótese $H_{0}$ para esse conjunto de números foi rejeitada. Na Figura 6 , é possível observar visualmente a discrepância entre as frequências relativas observadas e esperadas, encontrada pelo $X^{2}$ calculado.

Figura 6 - Gráfico das frequências relativas observadas e esperadas no caso dos EUA.

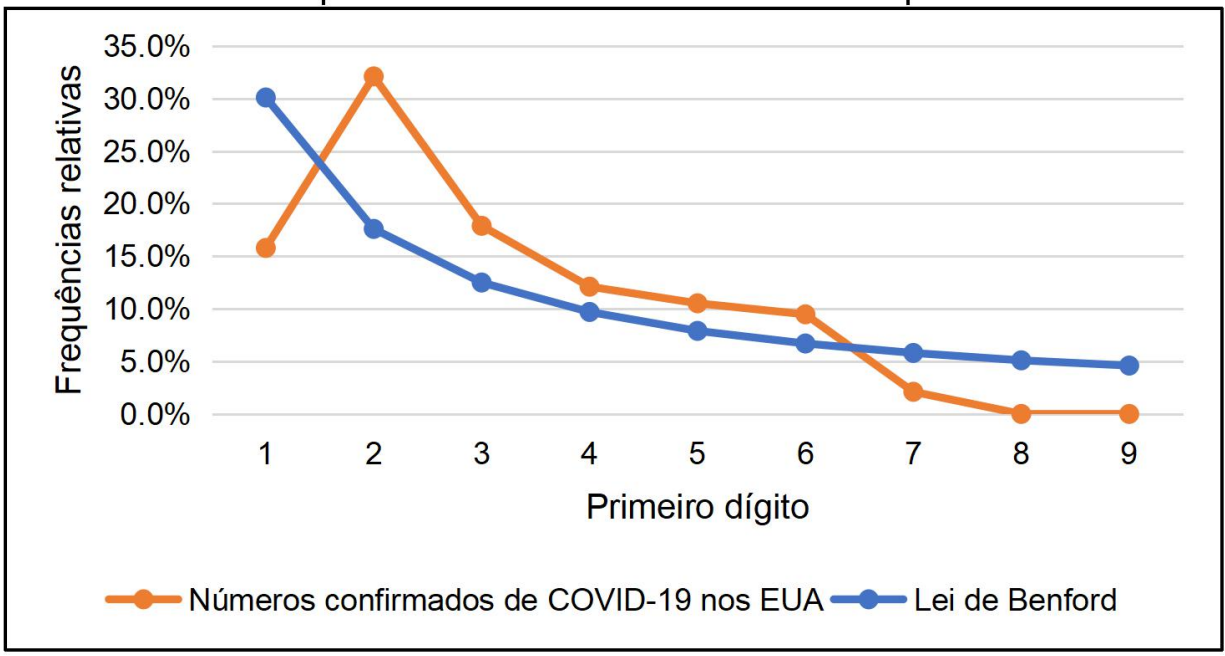

Fonte: Elaboração do autor para este texto (2020). 


\section{Conclusão}

O objetivo elencado neste artigo foi verificar se a Lei de Benford aplicava-se aos números de casos confirmados de COVID-19 em diferentes países (hipótese $H_{0}$ ). Ao utilizar o teste de comparação de frequências nos números de casos confirmados de COVID-19 na China, na Itália, na Nova Zelândia, no Brasil e nos EUA, foi possível avaliar a discrepância entre as frequências relativas observadas e as esperadas, para o aceite ou a rejeição da $H_{0}$, conforme organizado no Quadro 1.

Quadro 1 - Avaliação da hipótese H0 para os números de casos confirmados de COVID-19.

\begin{tabular}{|lccc|}
\hline Países & $\boldsymbol{X}^{2}$ calculado & $\boldsymbol{X}^{2}$ crítico & Hipótese $\boldsymbol{H}_{\mathbf{0}}$ \\
\hline${ }^{*}$ & 2,492 & 15,507 & Aceita \\
\hline & 8,157 & 15,507 & Aceita \\
\hline & 16,071 & 15,507 & Rejeitada \\
\hline
\end{tabular}

Fonte: Elaboração do autor (2020).

Assim, a hipótese $H_{0}$ de que a Lei de Benford se aplicava aos números de casos confirmados de COVID-19 foi aceita pelos números da China e da Nova Zelândia e rejeitada pelos números da Itália, do Brasil e dos EUA. A China, em particular, foi o país com os números que apresentaram a menor discrepância, seguida da Nova Zelândia. Por outro lado, a Itália passou por pouco do valor do $X^{2}$ crítico, enquanto o Brasil e os EUA foram os países com as maiores discrepâncias.

Em outros contextos, a Lei de Benford foi aplicada na auditoria de dados contábeis (NIGRINI, 1992, 2000, 2012), na auditoria de dados macroeconômicos (RAUCH et al., 2011) e até mesmo na busca de fraudes eleitorais (MEBANE, 2006, 2009). Com este estudo, não tivemos a intenção de auditar os números informados pelos países e contabilizados pela OMS. Segundo Campos, Wodewotzki e Jacobini (2013), é raro que frequências observadas concordem muito bem com as esperadas e, dessa forma, as discrepâncias encontradas nos números da Itália, do Brasil e dos EUA mostram que nem sempre a distribuição proposta pela Lei de Benford é a que melhor se ajusta para descrever o primeiro dígito. No entanto, com exceção dos números dos EUA, os outros países mostraram que o dígito 1 apareceu com mais frequência do que os demais.

Para futuras pesquisas, propomos investigar mais a fundo motivos que levaram os números de casos confirmados de COVID-19 a serem ajustados pela Lei de Benford, enquanto 
outros não. Talvez se deva adotar outras opções viáveis para tratar esses dados, estendendo a investigação para o segundo, o terceiro, o quarto dígito e assim por diante.

\section{Referências}

BENFORD, F. The law of anomalous numbers. Proceedings of The American Philosophical Society, v. 78, p. 551-572, 1938. Disponível em: https://www.jstor.org/stable/984802. Acesso em: 01 jul. 2020.

CAMPOS, R. C; WODEWOTZKI, M. L. L.; JACOBINI, O. R. Educação Estatística: teoria e prática em ambientes de modelagem matemática. Coleção Tendências em Educação Matemática. 2. ed. Belo Horizonte: Autêntica, 2013.

CARMO, H.; FERREIRA, M. M. Metodologia da investigação: guia para auto-aprendizagem. 2. ed. Lisboa-PT: Universidade Aberta, 2008.

CUNHA, F. C. R. da. Aplicações da lei Newcomb-Benford à auditoria de obras públicas. Orientador: Maurício Soares Bugarin. 2013. 486f. Dissertação (Mestrado em Regulação e Gestão de Negócios) - Universidade de Brasília, Brasília, 2013. Disponível em:

https://repositorio.unb.br/handle/10482/16379. Acesso em: 20 dez. 2020.

MEBANE, W. R. Election Forensics: vote counts and Benford's Law. Papers, Posters and Syllabi, The Society Political Methodology, n. 620, 2006.

MEBANE, W. R. Note on the presidential election in Iran. Michigan: University of Michigan, 2009.

NEWCOMB, S. Note on the frequency of use of the different digits in natural Numbers. American Journal of Mathematics, v. 4, n. 1, p. 39-40, 1881. DOI: https://doi.org/10.2307/2369148.

NIGRINI, M. J. The detection of income tax evasion through an analysis of digital frequencies. Tese (Ph.D.) - University of Cincinnati, Cincinnati, OH. 1992.

NIGRINI, M. J. Digital analysis using Benford's Law: Tests statistics for auditors. Global Audit Publication. Berlim, Heidelberg: Springer, 2000.

NIGRINI, M. J. Benford's law. Applications for Forensic Accounting, Auditing and Fraud Detection. Hoboken, New Jersey: John Wiley \& Sons, 2012.

OMS. World Health Organization. Disponível em: https://covid19.who.int/. Acesso em: 12 e 16 set. de 2020.

RAUCH, B.; BRÄHLER, G.; GÖTTSCHE, M.;ENGEL, S. Fact and fiction in EU-Governmental Economic Data. German Economic Review, v. 12, n. 3, p. 243-255, 2011. DOI:

https://doi.org/10.1111/j.1468-0475.2011.00542.x. 